\title{
Acoustic signature of laser shock peening for a qualitative evaluation of residual stresses
}

\author{
Saumyabrata Banerjee ${ }^{1}$ (C) P. Jonathan Phillips ${ }^{1}$. James Nygaard ${ }^{2} \cdot$ Paul D. Mason $^{1} \cdot$ Klaus Ertel $^{1}$. \\ Mariastefania De Vido ${ }^{1}$. Thomas Butcher ${ }^{1}$. Steph Tomlinson ${ }^{1}$. Jodie Smith ${ }^{1} \cdot$ Ric Allott $^{3}$. Chris Edwards ${ }^{1}$. \\ John Collier ${ }^{1}$
}

Received: 23 May 2019 / Accepted: 21 July 2019 / Published online: 31 July 2019

(c) The Author(s) 2019

\begin{abstract}
Acoustic signals recorded from laser shock peening (LSP) of aluminium, titanium and stainless steel alloys were analysed for different laser pulse widths, energy and confinement conditions. In this paper, we report that different materials and conditions registered unique acoustic signatures. Further, the acoustic signatures in the time domain were transformed to the frequency domain and were fitted to a lognormal distribution, yielding a unique set of parameters for each material and set of conditions. These parameters were benchmarked using the measurements of residual stress by the incremental centre hole-drilling (ICHD) method. Detailed analysis of the acoustic signal reveals that this method once calibrated with few ICHD data, can identify successful peening of materials.
\end{abstract}

\section{Introduction}

Peening of materials has being utilised for decades to improve material performance through the introduction of compressive residual stress, strain hardening and reduction in fatigue fracture. Advanced surface modification techniques, such as shot peening and laser shock peening (LSP) have shown significant improvement in the material performance. These techniques differ in the method for deformation of surface and depth of plastic zone. Shot peening uses multiple overlapping impacts of metal or ceramic shot on the workpiece to introduce compressive stress [1]. However, this technique introduces significant roughness on the surface and residual stress are limited in depth not exceeding $250 \mu \mathrm{m}$ [2]. Laser shock peening uses high energy, short pulse (typically ns pulses) to initiate the formation of plasma at the workpiece surface, which drives a plasma-induced pressure impulse into the workpiece. The pressure impulse travelling

Saumyabrata Banerjee

Saumyabrata.banerjee@stfc.ac.uk

1 Central Laser Facility, STFC Rutherford Appleton Laboratory, Didcot OX11 0QX, UK

2 Reaction Engines Ltd, Culham Science Centre, Abingdon OX14 3DB, Oxon, UK

3 Business and Innovation, STFC Rutherford Appleton Laboratory, Didcot OX11 0QX, UK through the workpiece gives rise to a layer of plastic deformation. LSP produces residual compressive stress magnitudes similar to that of conventional shot peening, however, the depth of the compressive stress typically exceeds beyond $1.0 \mathrm{~mm}$. Additionally, surface roughness is largely unaltered compared to shot peening. Numerical simulations for optimization and prediction of residual stresses have been performed by several groups [3-5] and effects of laser shock peening on residual stress and fatigue life have been reported by Dorman et al. [6]. Compressive stresses of up to $317 \mathrm{MPa}$ was reported in AA7075 T651 aluminum by Abeens et al. [7] utilizing LSP.

Owing to the advantages of LSP over conventional shot peening, it has become an indispensable tool for the material processing industry. LSP has been adopted by specialist sectors such as the aerospace and nuclear power generation industry where material performance is of critical concern. To increase the growth and development of LSP in the material processing industry, it is important to develop diagnostic techniques for in-situ and in-line monitoring and evaluation of the peening process. At present, the residual stresses in the material are measured using the incremental centre holedrilling (ICHD) technique or X-ray diffraction techniques [8, 9]. ICHD technique requires the use of three (or six) element strain gauge rosette, precision milling guide, gauge circuits and data-reduction relationships to deduce the principal residual stresses. This technique is destructive, expensive, 
time consuming and cannot be repeated for a large number of samples.

The sound generated by striking an object (hammering) has been used for decades as an indication of the quality (hardness), thickness or existence of defects in materials such as concrete in construction industry [10]. The advantage of this technique is its non-destructive nature. On the other hand, a lack of objectivity or reference is its disadvantage as it depends on an individual's experience and skills. Laser shock peening process can be considered an analogy to hammering a sample, were the laser pulse acts as a hammer (few ns in duration) followed by plastic deformation due to shock propagation (in few 10's of ns duration). LSP is also associated with generation of an acoustic signal (typically ms duration) owing to the ablation and bubble collapse in water confinement layer, often used to enhance compressive stress depth, as was demonstrated by Takata et al. [11]. Thus, once the sample surface obtains plastic deformation (in ns), reflected acoustic signal (in ms) can be used as a probe signal containing information regarding the permanently deformed quality of the surface. In case of LSP, the reference or benchmarking of the acoustic signal can be done with ICHD data.

In this paper, we report on an alternative approach of listening to and analysing the acoustic signals generated by the LSP process, which can be benchmarked against few ICHD data and can be used as an in-situ, inexpensive and in-line monitoring of industrial scale LSP process. We demonstrate that the acoustic signals recorded for different materials and conditions have unique signatures. Thus can be calibrated with ICHD data to identify successful peening of materials.

\section{Experimental setup and theory}

Laser shock peening experiments were carried out using the DiPOLE laser system at the Central Laser Facility in UK. DiPOLE is a scalable diode pumped, cryogenic gas cooled, multi-slab ceramic Yb:YAG laser system, capable of generating temporally shaped pulses (2-10 ns in duration) at $1030 \mathrm{~nm}$ wavelength up to $10 \mathrm{~J}$ at $10 \mathrm{~Hz}$ [12]. The output beam has a square $18 \mathrm{~mm}$ by $18 \mathrm{~mm}$ footprint. For all the experiments reported in this paper, the pulse shape was kept as a square flat-top pulse, and repetition rate was limited to $1 \mathrm{~Hz}$. The laser beam was image-relayed to preserve the spatial profile and was transported to the peening work station via a vacuum spatial filter (VSF) and set of $45^{\circ}$ mirrors coated for high reflection (HR) at $1030 \mathrm{~nm}$. Further, the beam was focused onto the workpiece using a $900 \mathrm{~mm}$ focal length lens. The workpiece was placed at a location from the lens where the spot size was $5 \mathrm{~mm}$ by $5 \mathrm{~mm}$ square. A thin layer of water $(\sim 4 \mathrm{~mm})$ was flowed over the surface of the workpiece to act as a confinement layer. A protective fused silica window [anti-reflection (AR) coated] was placed in front of the lens to protect it from water and debris ejecting from the workpiece during the LSP process. The whole area was enclosed by a $3 \mathrm{~mm}$ thick aluminium sheet to stop any scattering or stray reflections produced by the LSP process.

The workpiece was placed on an automatic XY-stage whose speed can be controlled by in-house software. This was used to synchronise the translation of the workpiece during LSP to control the overlapping of subsequent laser shots. A condenser microphone (Behringer C-4) was placed in the front of the workpiece to pick-up the acoustic signals reflected from the surface of the workpiece during the laser shots. The microphone had a signal-to-noise ratio of $75 \mathrm{~dB}$, maximum frequency response of $20 \mathrm{kHz}$ and a nominal impedance of $75 \Omega$. The output was connected to an oscilloscope (Agilent DSO9404A) for obtaining the temporal evolution of the LSP acoustic signal at a sampling rate of $100 \mathrm{MHz}$. Figure 1 shows the peening setup used for the experiments, also shown is a typical signal obtained from the LSP process. Previously, Takata et al. [11] demonstrated the relationship of detected AE waveforms and the LSP process by using a high speed camera and have observed multiple AEs owing to the laser ablation as well as the collapse of bubbles formed in the water confinement layer. The AE obtained in our setup constitutes not only the AEs generated due to the laser ablation and bubble collapse, but also the reflections from the peening enclosure. Thus forming a convoluted acoustic signal lasting 10's of milliseconds. Fourier transformation of such a convoluted signal to the frequency domain enabled analysis and benchmarking of the acoustic signal to the corresponding ICHD data.

The recorded acoustic signals were post-processed using Matlab software. The signal in the temporal domain was transformed to the frequency domain using Fast Fourier Transform (FFT) and was fitted with a lognormal distribution given by the equation:

$y=y_{0}+\frac{A}{\sqrt{2 \pi} \cdot \sigma \cdot x} \exp \left\{\frac{-\ln \left(\frac{x}{\mathrm{xc}_{\mathrm{c}}}\right)^{2}}{2 \cdot \sigma^{2}}\right\}$

where $A, \sigma$ and $\mathrm{Xc}$ are the area, standard deviation and mean frequency for the signal.

Figure 2 shows the FFT of the temporal signal obtained during Titanium (Ti6Al4V) LSP. It should be noted that multiple peaks exists in the plot (dashed black line), however, in this present work, we consider the dataset as unimodal and investigate the most dominant mode. Also shown is the fitted lognormal distribution (solid red line) yielding the parameters for the unique acoustic signal obtained from Titanium (Ti6Al4V) LSP. 


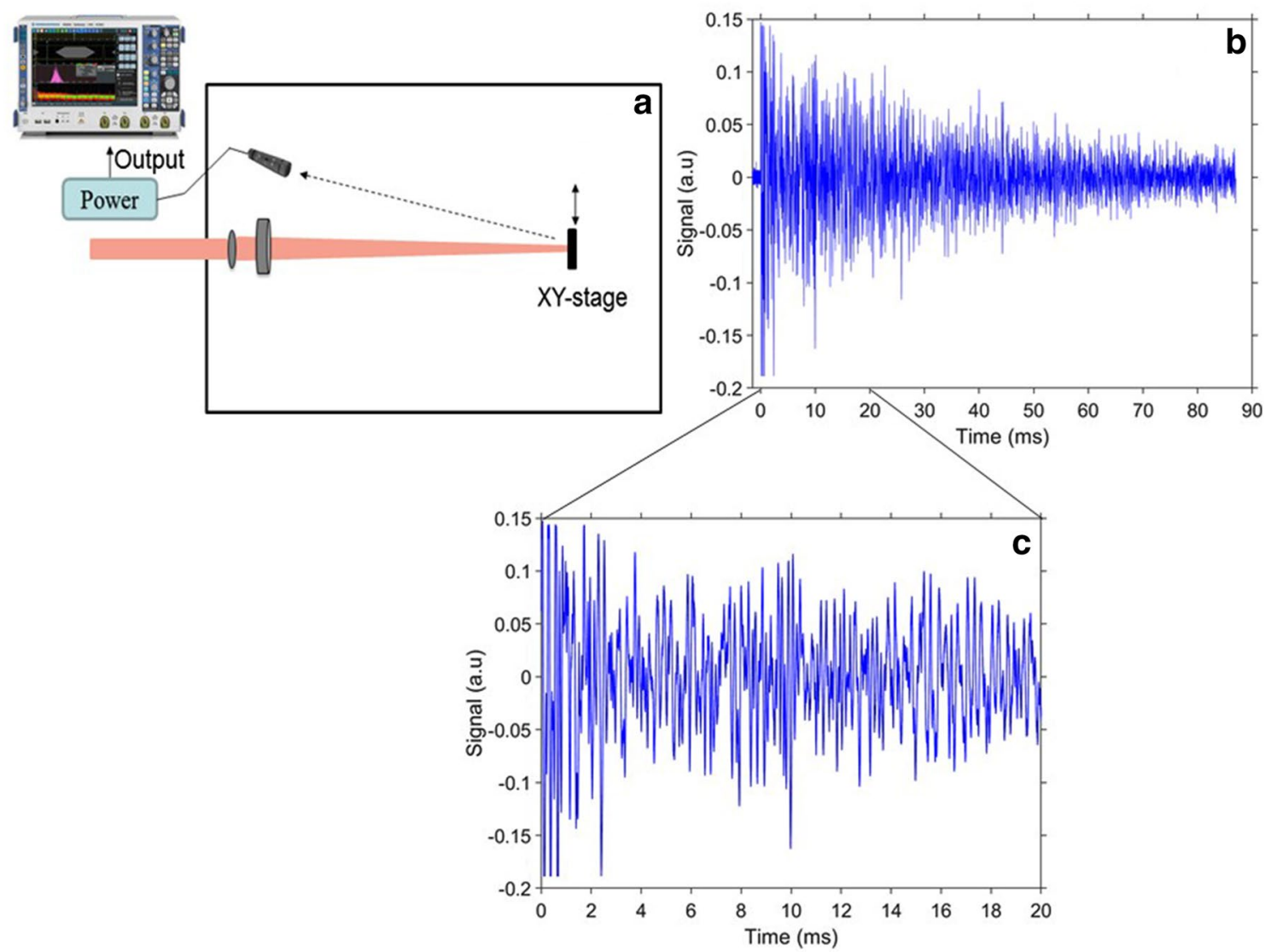

Fig. 1 a Layout of the LSP work station and b Typical temporal evolution of the LSP acoustic signal, $\mathbf{c}$ expanded view of the temporal signal showing convolution of multiple signals

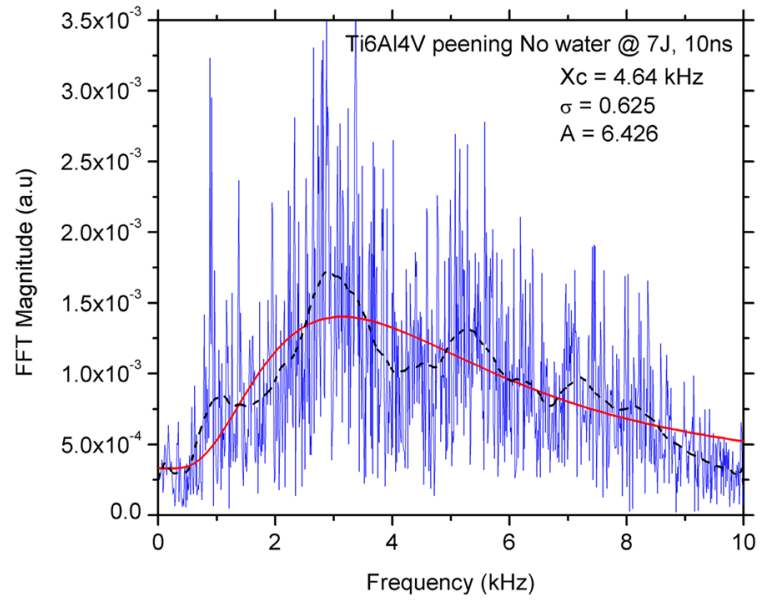

Fig. 2 FFT obtained from the temporal acoustic signal for Ti6Al4V with and without a confinement medium (water) at $7 \mathrm{~J}, 10 \mathrm{~ns}$. The red line shows a lognormal fit to the data. The dashed black line is the

The upper value for the frequency was restricted to $10 \mathrm{kHz}$ for all signals as the frequency response of the condenser microphone drops rapidly at high frequencies. Different sets of fitting parameters were produced for frequency

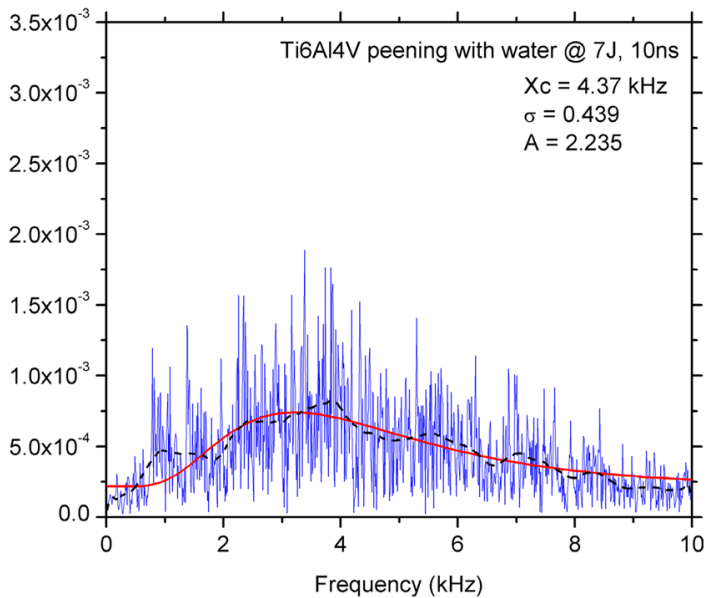

adjacent-averaged signal showing multiple peaks. Also given are the set of parameters obtained with the fit

data extending to $15 \mathrm{kHz}$, however, consistent results are found if the same range is used for all the signals. The upper value for the frequency was kept at $10 \mathrm{kHz}$ for all the data analysed for this manuscript. To simplify analysis, 
Fig. 3 Strain gauges installed on the peened sample and the arrangement for incremental drilling
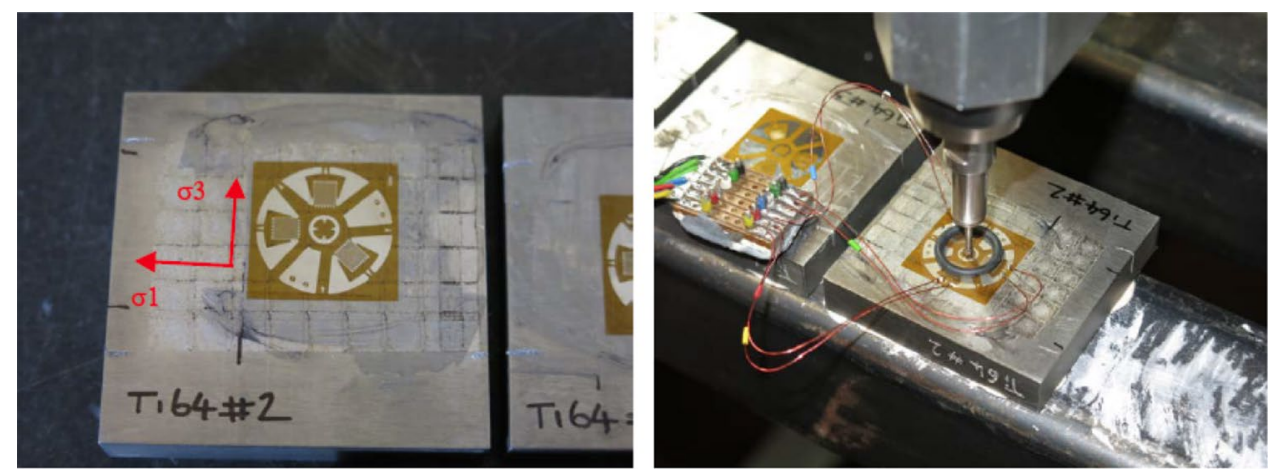

additional parameters $\delta=\xi / A$ where $\xi=\mathrm{Xc}(\mathrm{kHz}) \times \sigma$ were introduced.

The residual stresses of the peened materials were measured using the incremental centre hole-drilling (ICHD) technique at Cranfield University and Stresscraft Ltd. The strain gauge type, installation details, drilling cutters and hole diameters all comply with ASTM E837-13. Orbital hole drilling (circular milling) is carried out using tungsten carbide inverted cone cutters at a speed of $15,000 \mathrm{rpm}$ with a pre-set orbit eccentricity to give the required hole diameter. Figure 3 shows the gauges (EA-06-125RE-120) installed on the titanium sample and the arrangement for incremental drilling.

\section{Results and discussion}

Aluminium, titanium and stainless steel alloys were prepared by machining into dimensions of $50 \times 50 \times 15 \mathrm{~mm}$ and their surface polished to a roughness Ra of $0.9 \mu \mathrm{m}$. The samples were peened using the DiPOLE laser with different pulse energy ( $7 \mathrm{~J}$ and $4 \mathrm{~J}$ ) and duration (10 $\mathrm{ns}$ and $6 \mathrm{~ns}$ ) at $1 \mathrm{~Hz}$ repetition rate. The use of a transparent medium such as water on the surface of the workpiece acts as a confinement layer for the plasma and directs the shockwave deep into the material. Water was flowed on the target surface as a thin uniform confinement layer for the experiments reported in this manuscript. Two different confinement conditions, "WW" (with water) and "NW" (without water) were investigated. No coating was applied to the surface of the workpiece.

Three different aluminium alloys, namely A17075, Al7150 and Wire Arc Additive Manufacturing (WAAM) Al2319 (Department of manufacturing, University of Cranfield) were used as workpieces for the peening experiments. At first, to investigate the effect of multiple repeat shocks on the workpiece, the A17075 sample was peened at $7 \mathrm{~J}$, $10 \mathrm{~ns}$ corresponding to a peak intensity of $2.8 \mathrm{GW} / \mathrm{cm}^{2}$. The residual stresses for three different layers (i.e. number of shots at the same site) were measured using ICHD method. Figure 4 shows the measured residual stress along with the analysed data from the acoustic signal. As shown in Fig. 4a, the compressive stress as well as the depth of compression increases for the repeat laser shots (layers) on the workpiece, with a water confinement layer. The peak compressive stress increases from $-220 \mathrm{MPa}$ to $-275 \mathrm{MPa}$ and the depth increases from 1.0 to $1.8 \mathrm{~mm}$, respectively. Moreover, as shown in Fig. 4b, the compressive stresses are significantly less (10 times) in case of no water (NW) for the same energy and pulse duration laser shot.

As the acoustic signal reflects from the surface of the workpiece, any change in the surface residual stresses should also effect the reflected acoustic signal. A trend is seen in the analysed data from the acoustic signal (Fig. 4c), the parameter " $\delta$ " is constantly decreasing for the WW condition and tends to saturate after few shots, thus indicating that the level of compressive stresses no longer increase after 4-5 repeat shots on the workpiece. On the other hand, there is no overall reduction of the $\delta$-parameter for the no water $(\mathrm{NW})$ case, it shows an initial rise which may indicate an increase of tensile stresses at the surface of the workpiece due to the heat generated by laser ablation in the absence of the confinement layer. Unfortunately, ICHD data was not available for Al7075 in no water (NW) case.

Further, LSP experiments were done on WAAM-A12319 as well as on Al7150 to understand the effect of pulse energy for laser shock peening of aluminium alloys. Figure 5a shows the residual stresses measured by ICHD method for WAAM-Al2319 at $7 \mathrm{~J}, 6 \mathrm{~ns}$ and $4 \mathrm{~J}, 6 \mathrm{~ns}$ for WW and NW cases, Fig. $5 \mathrm{~b}$ shows the parameters analysed by the corresponding acoustic signal obtained during the LSP experiments. In NW case, it is evident from Fig. 5a that the tensile stress increases as the laser energy is increased from 4 to $7 \mathrm{~J}$ at $6 \mathrm{~ns}$ pulse duration. This is in agreement with the increase in the $\delta$-parameter in Fig. 4c for repeated laser shocks at the same energy. Figure $5 \mathrm{~b}$ shows the parameters ( $A$ and $\sigma$ ) from the analysed acoustic signals generated during LSP for different energy and confinement conditions. Note that a clear demarcation is visible with the WW data being towards 

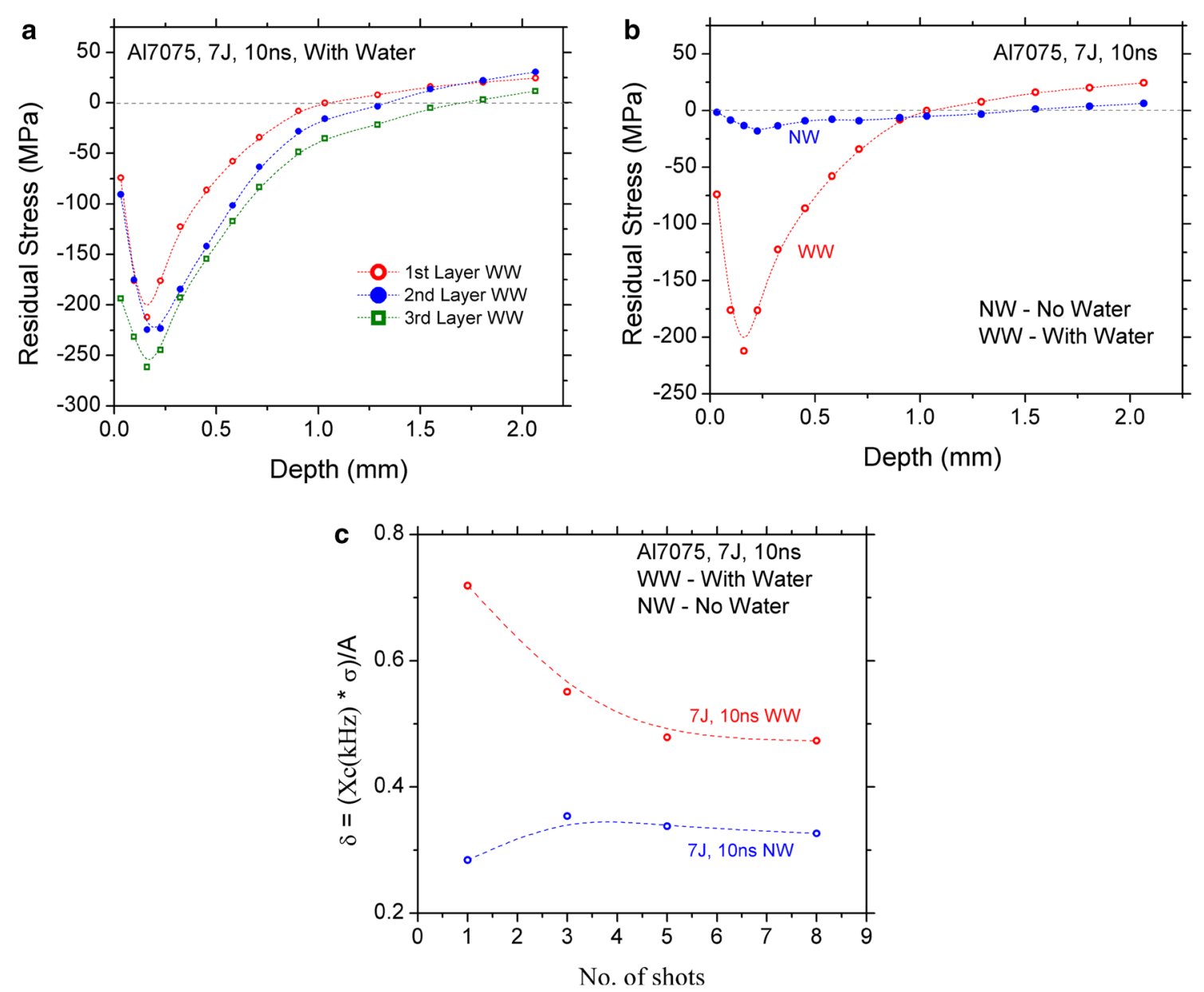

Fig. 4 a Compressive stress measured using ICHD method for three repeat shocks with water in aluminium (A17075). b Compressive stress measured for different confinement conditions at $7 \mathrm{~J}, 10 \mathrm{~ns}$ and c post-processed data of the acoustic signal. Red dotted line is for the WW condition and blue dotted line is for the NW condition
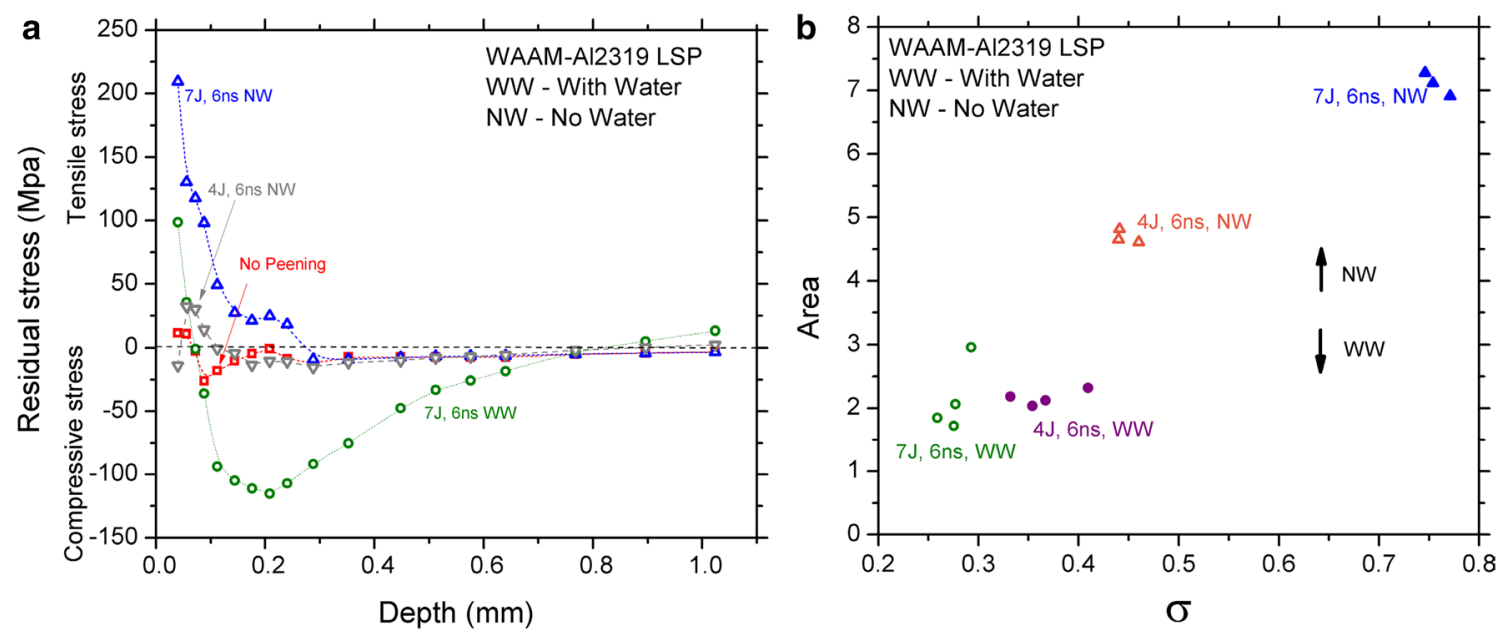

Fig. 5 a Residual stress measured by ICHD method for WAAM-A12319 for different laser energy, b plots of fitting parameters analysed from the acoustic signals in the different LSP experiments 


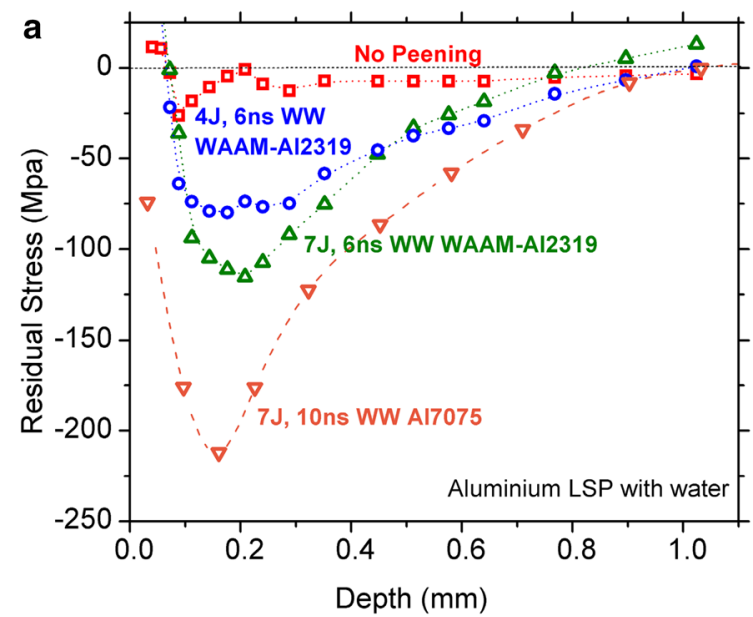

Fig. 6 a Compressive stress measured by ICHD method for different laser energy, pulse width and aluminium alloys with water (WW) as a confinement layer, $\mathbf{b}$ plot of fitting parameters (Xc and $\sigma$ ) analysed

the lower side of the plot and the NW data towards the top. Thus, acoustic signals can clearly identify the two different confinement cases. Figure 6 a shows the compressive stress for the WAAM-Al2319 as well as for Al7075 at different energy and pulse width when water was used as confinement layer.

Figure $6 \mathrm{~b}$ shows the parameters (Xc and $\sigma$ ) for different aluminium alloys, laser energy and pulse width for LSP with water (WW). Note that different aluminium alloys (Al7075 and A17150) register different acoustic signals (yielding unique fitting parameters) for the same laser energy and pulse width ( $7 \mathrm{~J}, 10 \mathrm{~ns})$. Moreover, most data obtained from different energy and pulse width sit on the $\xi=2$ line. Comparing Figs. 4a and 6b for Al7075 (for repeat shocks), it seems that higher compressive stress in the material pushes the data points towards $\xi=1$ line, Moreover, with increase in the compressive stress, both Xc as well as $\sigma$ decreases as seen for WAAM-Al2319 (comparing Fig. 6a and b). No ICHD data showing compressive stress was available for A17150 laser peened sample because of a drill failure during measurement.

In the next series of experiments, WAAM-Ti6Al4V alloy (Department of manufacturing, University of Cranfield) was used as the workpiece for LSP. As for the aluminium alloys, WAAM-Ti6Al4V alloy was subjected to different energy, pulse width and confinement conditions. Figure 7 shows the results obtained from ICHD method as well as the results from acoustic signal analysed during titanium laser shock peening. Figure 7 a shows the ICHD measured residual stresses for different laser energy at $6 \mathrm{~ns}$ with different confinement conditions. A decrease of compressive stress (increase of tensile stress) is obtained with the increase of laser energy without water (NW) at the surface of the

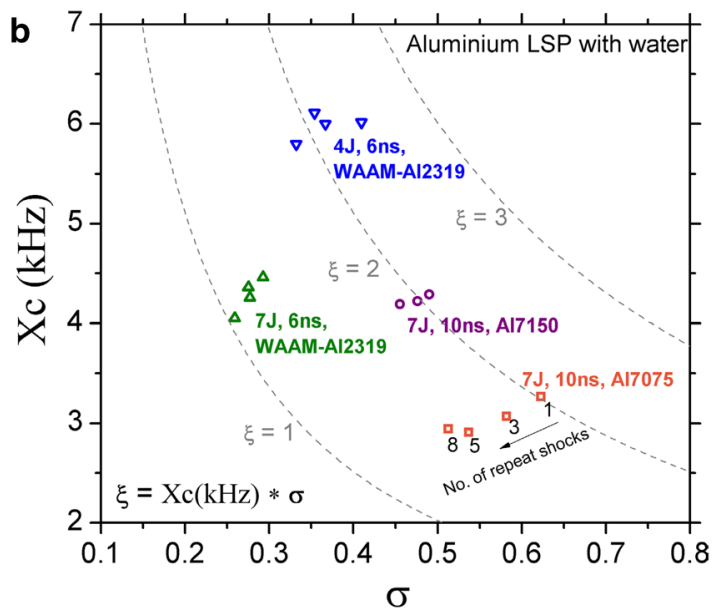

from the acoustic signals in the different LSP experiments. Gray dotted lines are contour lines for multiplication of the y-axis with the $\mathrm{x}$-axis for $\xi=1,2$ and 3

workpiece. Increase in the compressive stress is seen at $7 \mathrm{~J}$, $6 \mathrm{~ns}$ laser parameters with water (WW) and peak value of compressive stress reaches $-290 \mathrm{MPa}$. However, the depth of compressive stress is less in titanium alloy compared to aluminium alloy. Figure $7 \mathrm{~b}$ shows the parameters $(A$ and $\sigma$ ) analysed by the generated acoustic signal in the Ti-LSP experiments. As seen in the previous experiments with aluminium, a clear separation is again seen in the data for different confinement conditions. Experiments done with water (WW) are at the bottom of the plot whereas the experiments without water (NW) are towards the top. The repeatability of the trends in the analysed data both for aluminium and titanium LSP has given confidence on the method used to analyse the acoustic signals. Figure $7 \mathrm{c}$ shows the parameters (Xc and $\sigma$ ) for Ti-LSP with water, the data points lie on or near the $\xi=2$ line as in the case of Al-LSP.

Finally, to confirm our hypothesis regarding the increase of $\delta$-parameter showing an increase in the tensile stress at the sample surface, we experimented on horizontally grown 3D printed stainless steel SS316L. LSP experiments were carried out at $7 \mathrm{~J}, 10 \mathrm{~ns}$ for different confinement conditions. Figure 8a shows the ICHD measured residual stress in SS316L peened with different confinement condition and using multiple laser shocks at the same site. Figure $8 \mathrm{~b}$ shows the data obtained from the analysed acoustic signals from different materials (A17075, SS316L and Ti6Al4V) at $7 \mathrm{~J}$, $10 \mathrm{~ns}$ LSP without water (NW).

Note that the ICHD measured residual stress in SS316L show an increase of tensile stress at the surface from 75 to $230 \mathrm{MPa}$ for two repeat shocks at $7 \mathrm{~J}, 10 \mathrm{~ns}$ without water. Data analysed from the acoustic signal for the same sample (SS316L) shows an increase in the $\delta$-parameter for the second repeat shock, validating the hypothesis that for repeat 


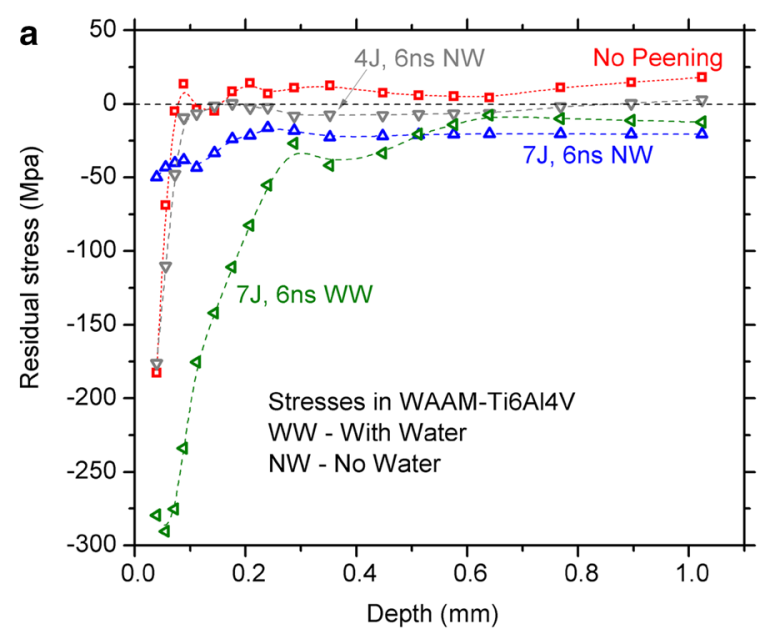

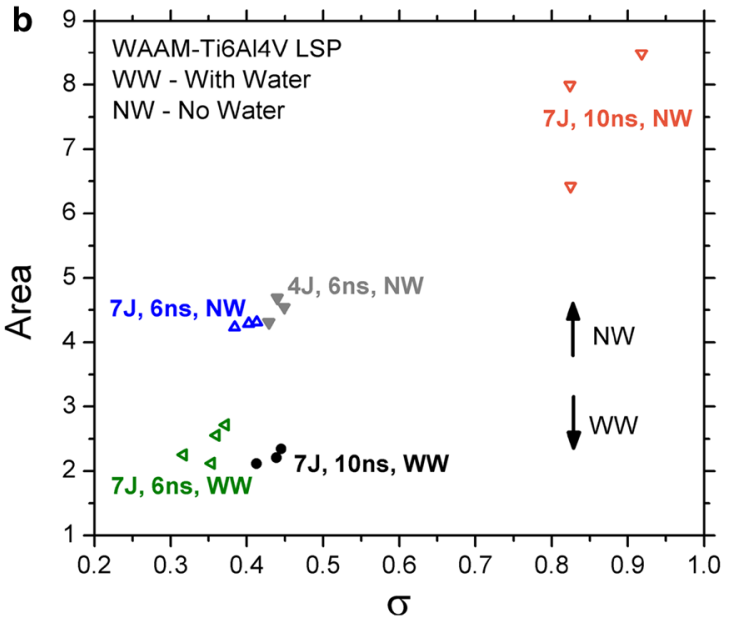

Fig. 7 Measurements of residual stress from ICHD method and the results from the analyses of the acoustic signals generated during the Ti6Al4V laser shock peening process at different energy, pulse width

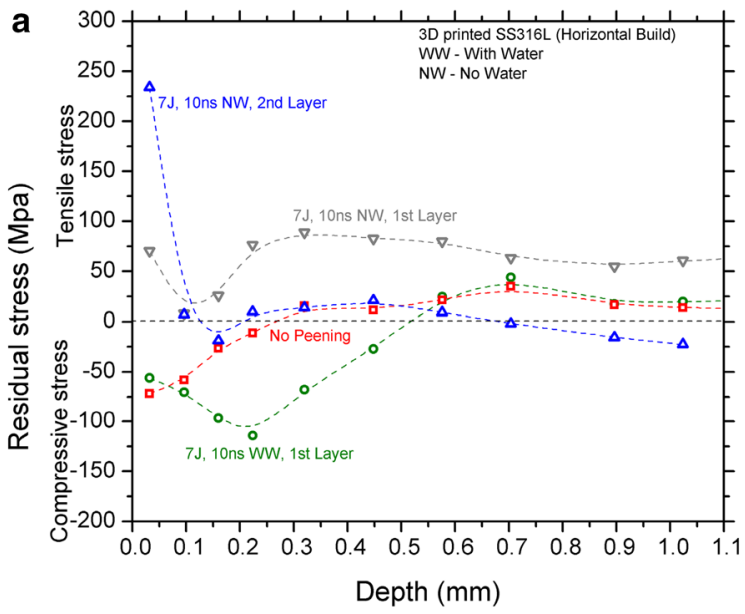

Fig. 8 a ICHD measured residual stress for SS316L LSP at $7 \mathrm{~J}, 10 \mathrm{~ns}$ with water (WW) and without water (NW), also shown is the measured stress for the 2 nd repeat shock without water (NW) in the same

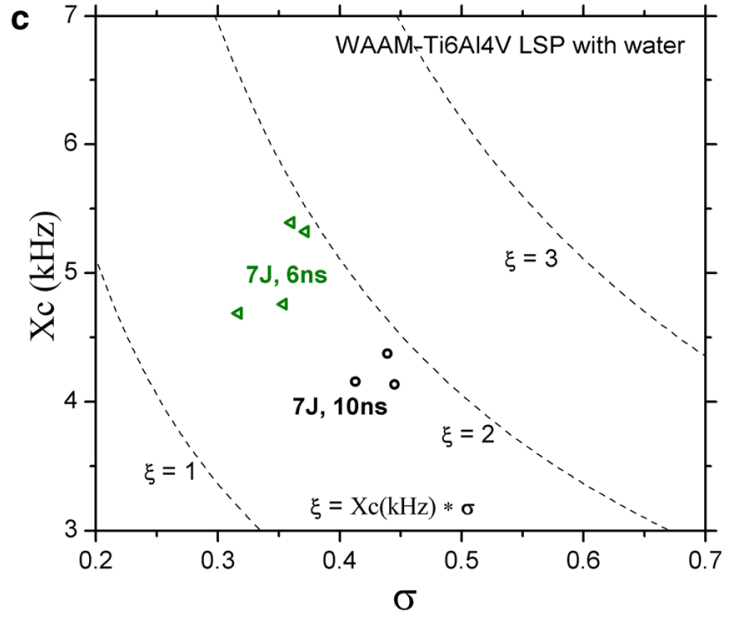

and confinement conditions. Gray dotted lines are contour lines for multiplication of the $\mathrm{y}$-axis with the $\mathrm{x}$-axis for $\xi=1,2$ and 3

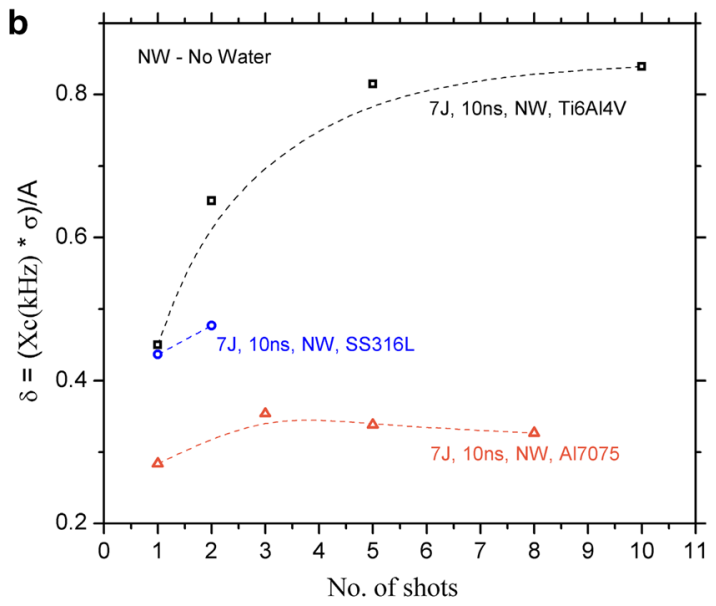

sample. b $\delta$-parameter from the acoustics signals during LSP from A17075, SS316L and Ti6A14V 
laser shocks, an increase in the $\delta$-parameter indicate increase of tensile stress and a decrease show an increase of compressive stress on the sample surface. LSP without water on WAAM-Ti6Al4V at $7 \mathrm{~J}, 10 \mathrm{~ns}$ show significant increase of $\delta$-parameter compared to SS316L and Al7075. Unfortunately, no ICHD data was available to compare this result, however, as is shown for the $\mathrm{SS} 316 \mathrm{~L}$ sample, an increase in the tensile stress at the sample surface is expected in the WAAM-Ti6Al4V sample as well.

\section{Conclusion}

In summary, we demonstrate that the acoustic signals generated during LSP process contains information regarding the surface of the peened sample depending upon the material, alloy, laser energy, pulse width and confinement conditions. It was found that a decrease of the $\delta$-parameter of the analysed acoustic signal indicate an increase of the compressive stress and reverse indicates an increase of tensile stress during multiple repeat shocks in LSP of the same material. Further, most of the data collected for with water (WW) LSP of different materials, decrease of both Xc as well as $\sigma$ indicate increase of the compressive stress. As the acoustic signals generated during laser shock peening have a unique signature, they can be calibrated with few ICHD data to identify successful peening of materials and have the potential to be used as an in-situ, inexpensive and in-line process monitoring diagnostics for industrial scale LSP processes.

Open Access This article is distributed under the terms of the Creative Commons Attribution 4.0 International License (http://creativeco mmons.org/licenses/by/4.0/), which permits unrestricted use, distribution, and reproduction in any medium, provided you give appropriate credit to the original author(s) and the source, provide a link to the Creative Commons license, and indicate if changes were made.

\section{References}

1. M. Kobayashi, T. Matsui, Y. Murakami, Mechanism of creation of compressive residual stress by shot peening. Int. J. Fatigue 20, $351(1998)$
2. Harold Luong, Michael R. Hill, The effects of laser peening and shot peening on high cycle fatigue in 7050-T7451 aluminium alloy. Mat. Sci. Eng A 527, 699 (2010)

3. M.I. Hatamleh, J.S. Mahadevan, A.S. Malik, D. Qian, Variable damping profiles for laser shock peening simulation using modal analysis and the SEATD method. In: ASME 2017 12th international manufacturing science and engineering conference, American Society of Mechanical Engineers, 2017, pp. V001T02A032-V001T02A032

4. R.A. Brockman, W.R. Braisted, S.E. Olson, R.D. Tenaglia, A.H. Clauer, K. Langer, M.J. Shepard, Prediction and characterization of residual stresses from laser shock peening. Int $\mathrm{J}$ Fatigue 36, 96-108 (2012)

5. M. Frija, M. Ayeb, R. Seddik, R. Fathallah, H. Sidhom, Optimization of peened-surface laser shock conditions by method of finite element and technique of design of experiments. Int. J. Adv. Manuf. Technol. 97, 1-19 (2018)

6. M. Dorman, M.B. Toparli, N. Smyth, A. Cini, M.E. Fitzpatrick, P.E. Irving, Effect of laser shock peening on residual stress and fatigue life of clad 2024 aluminium sheet containing scribe defects. Mater. Sci. Eng. A 548, 142-151 (2012)

7. M. Abeens, R. Muruganandhan, K. Thirumavalanvan, S. Kalainathan, Surface modification of AA7075 T651 by laser shock peening to improve the wear characteristics. Mater. Res. Express 6(6), 066519 (2019)

8. A. Niku-Lari, J. Lu, J.F. Flavenot, Measurement of residual-stress distribution by the incremental hole-drilling method. J. Mech. Work. Tech. 11, 167 (1985)

9. https://www.vishaypg.com/docs/11053/tn503.pdf, Document Number: 11053, 19 (2010)

10. A. Yamashita, T. Hara, T. Kanuko, Hammering test with integration of image and sound signal processing. J. Environ. Eng. 3(2), 363 (2008)

11. T. Takata, M. Enoki, P. Chivavibul, A. Matsui, Y. Kobayashi, Acoustic emission monitoring of laser shock peening by detection of underwater acoustic wave. Mater. Trans. 57(5), 674-680 (2016)

12. S. Banerjee, K. Ertel, P.D. Mason, P.J. Phillips, M. De Vido, J.M. Smith, T.J. Butcher, C. Hernandez-Gomez, R.J.S. Greenhalgh, J.L. Collier, DiPOLE: a $10 \mathrm{~J}, 10 \mathrm{~Hz}$ cryogenic gas cooled multislab Yb:YAG laser. Opt. Express 23, 19542 (2015)

Publisher's Note Springer Nature remains neutral with regard to jurisdictional claims in published maps and institutional affiliations. 